\title{
Comparison of the Chemical Components of Powdered Green Tea Sold in the US
}

\author{
Hideki HORIE ${ }^{1 *}$, Kaori EMA ${ }^{1}$, Hiroshi NISHIKAWA ${ }^{2}$ and Yoriyuki NAKAMURA ${ }^{3}$ \\ ${ }^{1}$ Institute of Fruit Tree and Tea Science, National Agriculture and Food Research Organization \\ (Shimada, Shizuoka 428-8501, Japan) \\ ${ }^{2}$ Tea Industry Development Division, Shizuoka Prefectural Government (Shizuoka, Shizuoka 420- \\ 8601, Japan) \\ ${ }^{3}$ Tea Science Center, University of Shizuoka (Shizuoka, Shizuoka 422-8526, Japan)
}

\begin{abstract}
The international market for green tea is growing. Samples of twenty-five powdered green teas were collected from the market in the United States, and their chemical components were compared. Their prices varied widely, from $\$ 4.3$ to $\$ 160$ per $100 \mathrm{~g}$. The higher-priced teas were labeled as "matcha" and are used in the traditional Japanese tea ceremony. They showed a higher content of chlorophyll and of free amino acids (theanine and arginine) than the less expensive teas. They also showed a lower content of epigallocatechin as well as a lower conversion percentage of chlorophyll to pheophytin than those of lower prices. Matcha is traditionally produced in Japan and considered one of the country's most precious teas. In spite of the large differences in chemical composition compared with the highly priced powdered green teas (ceremonial-grade matcha), the cheaper teas are also labeled as "matcha" in the US market. It is, therefore, necessary to come up with an international definition of matcha to resolve the confusion.
\end{abstract}

Discipline: Tea industry

Additional key words: chlorophylls, catechins, theanine, matcha

\section{Introduction}

Several varieties of green tea are produced in Japan where "sencha" is the most popular type, while "gyokuro" is a high-grade tea produced from shade-grown tea leaves. Both sencha and gyokuro are leaf-type teas, but powdered green teas have become more popular (Kuwabara 2016) because they require no "kyusu" (a special pot to infuse tea from leaf-type green teas) and all the nutrients can be imbibed when using powdered teas. Powdered green teas are also used as ingredients for cooking and as raw materials in the food industry. Among powdered teas, matcha is a specialty tea used in the traditional Japanese tea ceremony, and the leaves of matcha are harvested after long-term shade treatment of the tea bushes.

Meanwhile, the international market for green tea is growing: the world trade of green teas increased from less than $100,000 \mathrm{t}$ in 1990 to more than $350,000 \mathrm{t}$ in 2013 (Japan Tea Central Public Interest Incorporated
Association). Tea exports from Japan have also increased rapidly from 300 to 3,000 $t$ during the same period (Trade Statistics of Japan, Ministry of Finance). The United States is the largest importer of tea from Japan. While we do not have any reliable statistical data on the types of teas exported from Japan, a fairly significant amount of powdered teas is estimated as being imported to the US from Japan. Other tea-producing countries also export green teas to the US, and some of these exports probably include powdered teas. It would therefore be of great interest to collect powdered teas in the US and compare their quality.

It is known that high-grade Japanese green teas including matcha are rich in amino acids, especially theanine and arginine (Goto et al. 1996). Matcha is cultivated in the shade, which is known to change the constitution of catechins (Matsunaga et al. 2016) and increase the content of chlorophylls (Kohata et al. 1999). We analyzed the contents of amino acids, catechins, chlorophylls, and pheophytins in powdered tea samples using high-performance liquid

This paper reports the results obtained in a project conducted by the NARO Bio-oriented Technology Research Advancement Institution (entitled "The Special Scheme to Create Dynamism in Agriculture, Forestry and Fisheries through Deploying Highly Advanced Technology").

*Corresponding author: e-mail horie@affrc.go.jp

Received 27 March 2017; accepted 5 July 2017. 
Table 1. Powdered tea samples collected in the US

\begin{tabular}{llllrrr}
\hline \hline \multicolumn{1}{c}{ Label } & Country* & Organic & Price US\$ & Package $(\mathrm{g})$ & $\$ / 100 \mathrm{~g}$ \\
\hline A & Matcha, ceremonial & Japan & & 48.00 & 30 & 160.0 \\
B & Matcha, ceremonial & Japan & O** & 37.00 & 25 & 148.0 \\
C & Matcha, ceremonial & Japan & $\bigcirc$ & 28.00 & 28 & 100.0 \\
D & Matcha & Japan & & 78.14 & 100 & 78.1 \\
E & Matcha, ceremonial & Japan & $\bigcirc$ & 16.50 & 25 & 66.0 \\
F & Matcha, ceremonial & Japan & & 18.08 & 28 & 64.6 \\
G & Matcha, ceremonial & Japan & $\bigcirc$ & 19.95 & 40 & 49.9 \\
H & Matcha, culinary & Japan & $\bigcirc$ & 35.77 & 100 & 35.8 \\
I & Matcha, ceremonial & Japan & $\bigcirc$ & 10.28 & 30 & 34.3 \\
J & Matcha & Japan & & 8.00 & 28 & 28.6 \\
K & Matcha & Japan & $\bigcirc$ & 26.99 & 99 & 27.3 \\
L & Green tea powder & China & $\bigcirc$ & 25.00 & 100 & 25.0 \\
M & Matcha & unknown & $\bigcirc$ & 24.95 & 100 & 25.0 \\
N & Green tea powder & Japan & $\bigcirc$ & 11.50 & 50 & 23.0 \\
O & Matcha & China & $\bigcirc$ & 19.21 & 84 & 22.9 \\
P & Matcha & China & $\bigcirc$ & 8.99 & 50 & 18.0 \\
Q & Matcha, culinary & unknown & $\bigcirc$ & 24.95 & 141 & 17.7 \\
R & Matcha, culinary & Japan & $\bigcirc$ & 3.00 & 25 & 12.0 \\
S & Matcha & Japan & & 51.38 & 498 & 10.3 \\
T & Matcha & Korea & $\bigcirc$ & 18.99 & 200 & 9.5 \\
U & Matcha & China & $\bigcirc$ & 24.71 & 283 & 8.7 \\
V & Matcha, drink & Japan & $\bigcirc$ & 26.24 & 311 & 8.4 \\
W & Matcha & Taiwan & & 18.99 & 272 & 7.0 \\
X & Matcha & Japan & $\bigcirc$ & 26.49 & 500 & 5.3 \\
Y & Matcha & Taiwan & & 10.75 & 250 & 4.3 \\
\hline
\end{tabular}

*: country of the production of the tea leaves

**: labeled as organic products

chromatography (HPLC).

\section{Materials and methods}

We collected 25 powdered tea samples, mostly online sales items, sold in the US in the autumn of 2014. The components of these samples were analyzed in less than four months after the samples were obtained. We used a HPLC system (LC10ADvp, Shimadzu, Kyoto, Japan) for the analysis. First, we determined the contents of chlorophyll $a$ (Chl $a$ ), chlorophyll $b$ (Chl $b$ ), pheophytin $a$ (Phy $a$ ), and pheophytin $b$ (Phy $b$ ) according to the method presented by Kohata et al. (1998) using the system, as these pigments easily deteriorate (Haraguchi 2002). The conversion percentage of chlorophyll to pheophytin was calculated according to Kohata et al. (1999) using the analytical results. Next, the levels of four major catechins - epigallocatechin gallate (EGCG), epigallocatechin (EGC), epicatechin gallate (ECG), and epicatechin (EC) - were measured according to the method proposed by Maeda-Yamamoto et al. (2004). Theanine and other free amino acids were analyzed according to our previous report (Horie 2012) using HPLC, but the fluorescence-derivatization step was performed manually.

\section{Results}

\section{Samples}

Table 1 lists the collected powdered tea samples in order of the price per $100 \mathrm{~g}$, along with the country of production and size of the package. Of the 25 samples, 16 were imported from Japan, four were from China, two were from Taiwan, one was from Korea, and the remaining two samples were of indeterminable origin. The prices of the samples collected varied widely, from $\$ 4.3$ to $\$ 160$ per $100 \mathrm{~g}$. In Japan, matcha is a precious tea used in the traditional tea ceremony and low-grade teas are simply called powdered green teas. However, even the cheaper teas (W, X, and Y) are sold as matcha in the US. The most expensive teas (over $\$ 30$ per $100 \mathrm{~g}$ ) were produced in Japan, with most of these teas being labeled ceremonial-grade matcha. A few medium-grade teas were labeled as culinary-grade matcha. Organic tea is not always popular in Japan, although 16 of the 25 samples were labeled as "organic" (including 11 of the 16 samples imported from Japan).

Sample V was labeled as a "matcha drink" and its lev- 


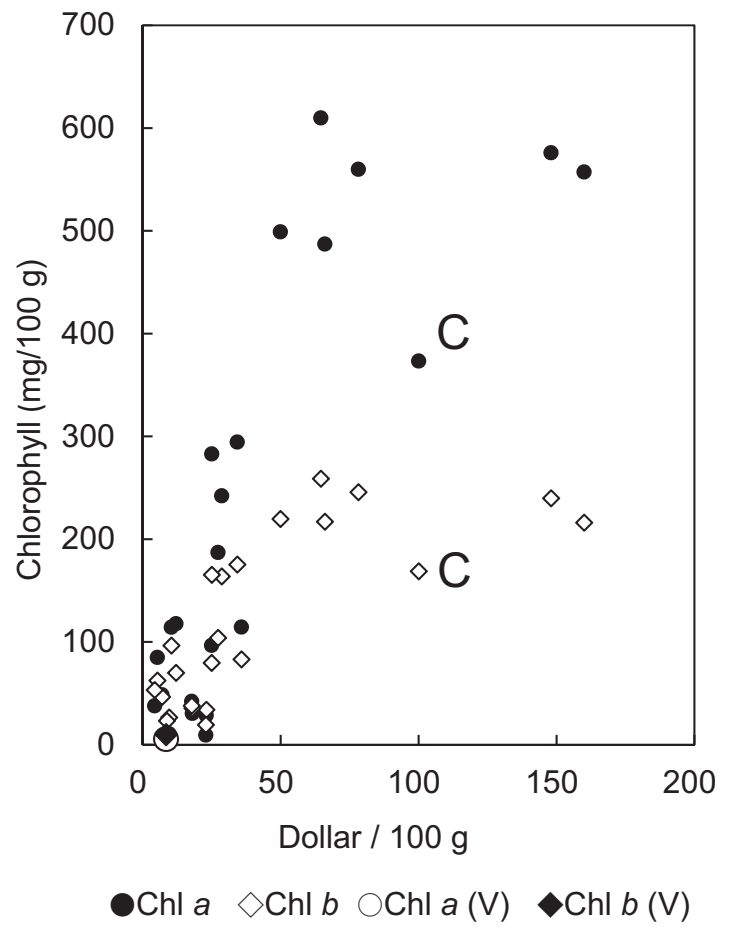

Fig. 1. Chlorophyll content in powdered green teas "C" added to or $\diamond$ indicates the content of sample C.

els of such chemical components as chlorophylls, catechins and amino acids (Figs. 1, 3 and 4) were lower than those of other similarly priced samples. It was labeled as having brown rice added as an ingredient. Therefore, sample V was treated as an exception in this report.

\section{Comparison of the Components}

(1) Chlorophylls and related compounds

There are no official chemical requirements to grade powdered green teas in Japan, but the green color of the tea powder is important for high-grade matcha. Kohata et al. (1999) showed that matcha contained higher amounts of chlorophyll $a$ and $b$ than sencha, and that higher-grade matcha showed a lesser conversion percentage of chlorophyll to pheophytin than that of lower-grade samples. The contents of chlorophylls and pheophytins in the samples collected were determined. Figure 1 shows the content of Chl $a$ and $\mathrm{Chl} b$. There was a tendency for more expensive samples to possess higher amounts of $\mathrm{Chl} a$ and $\mathrm{Chl} b$, as the samples costing more than $\$ 50$ per $100 \mathrm{~g}$ contained more than $370 \mathrm{mg} / 100 \mathrm{~g}$ of Chl $a$ and $160 \mathrm{mg} / 100 \mathrm{~g}$ of Chl $b$, while the samples costing less than $\$ 20$ contained less than $120 \mathrm{mg} / 100 \mathrm{~g}$ of the respective chlorophylls.

Figure 2 shows the conversion percentages of chlorophyll to pheophytin. The conversion percentages of the samples costing more than $\$ 50$ per $100 \mathrm{~g}$ were less than

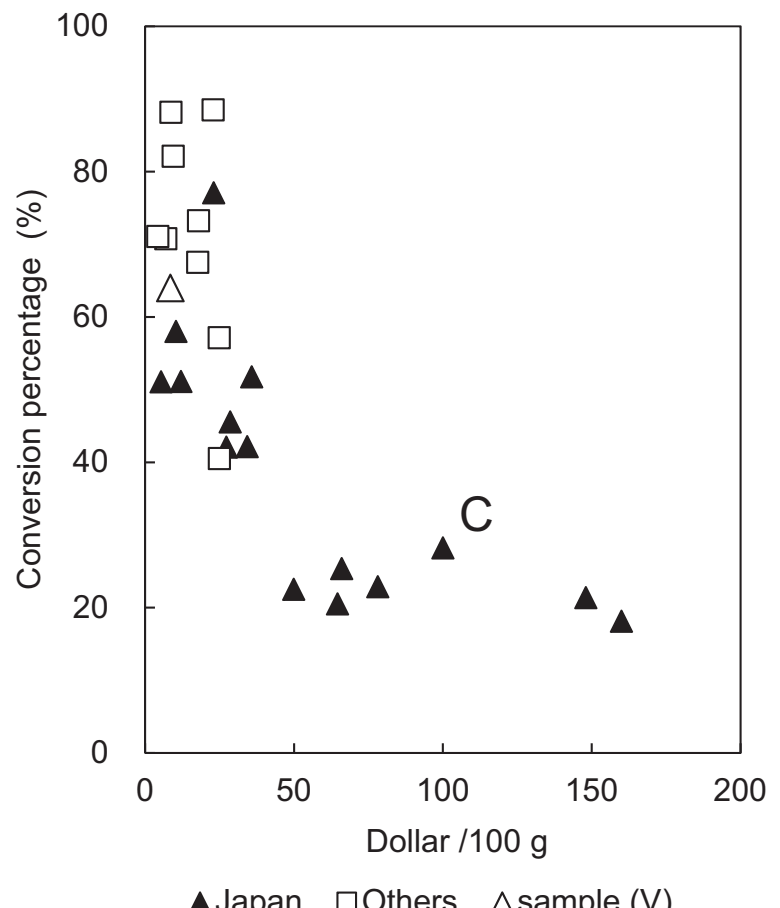

Fig. 2. Conversion percentage of chlorophyll to pheophytin $\boldsymbol{\Delta}$ : Teas produced in Japan $\square$ : Teas produced in countries other than Japan.

$30 \%$, while those costing less than $\$ 20$ were more than $50 \%$. As shown in this figure, the countries of production of the tea leaves were also examined. The samples produced in Japan tended to show lower conversion percentages than similarly priced samples produced in other countries.

(2) Catechins and free amino acids

Catechins are substances related to the bitterness and astringency of tea. Figure 3 shows a comparison of the levels of the two major catechins in tea (EGCG and EGC). The EGC content increases with aging of the new shoots, while shade treatment suppresses the rise of EGC (Matsunaga et al. 2016). Thus, the EGC content is expected to be low in high-grade matcha, which is produced from new shoots under long-term shade treatment. EGC levels varied between 1.0 and $4.6 \mathrm{~g} / 100 \mathrm{~g}$ in our samples, whereas the samples costing more than $\$ 50$ per $100 \mathrm{~g}$ contained less than $2.3 \mathrm{~g} / 100 \mathrm{~g}$, with one exception (sample C). The EGCG content varied between 5.3 and $9.3 \mathrm{~g} / 100 \mathrm{~g}$, whereas the samples costing more than $\$ 50$ contained between 5.9 and $7.1 \mathrm{~g} / 100 \mathrm{~g}$.

Amino acids contribute to the umami taste of tea, and the most abundant amino acids in high-grade matcha are theanine and arginine (Goto et al. 1996). Figure 4 shows the levels of these amino acids. The more expensive teas showed higher levels of both amino acids; the levels of theanine and arginine in the samples costing more than $\$ 50$ 


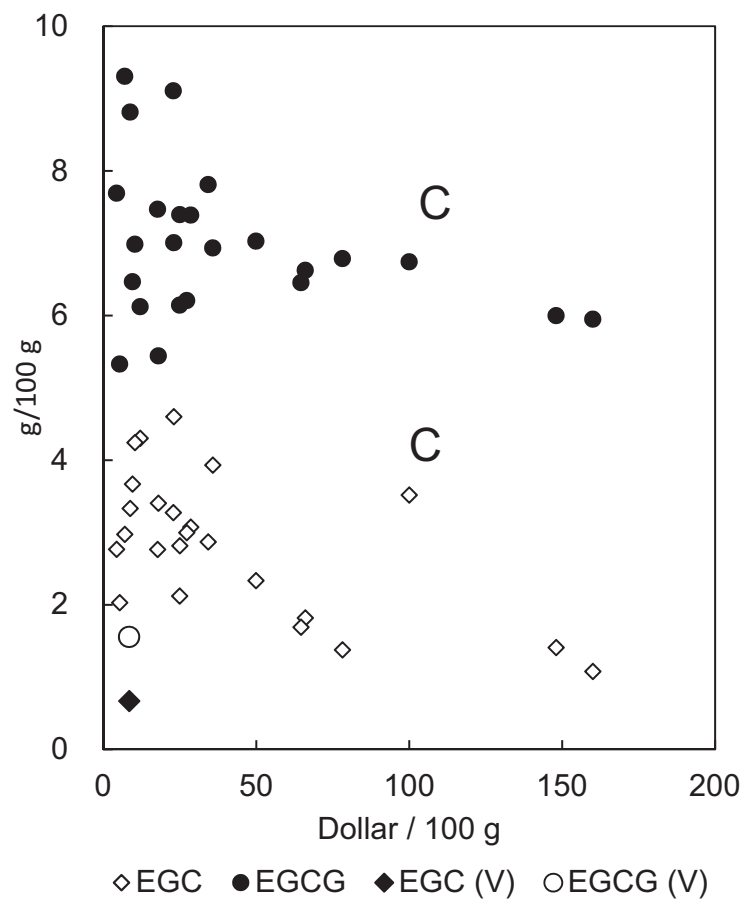

Fig. 3. EGC and EGCG contents in powdered green teas EGC: epigallocatechin; EGCG: epigallocatechin gallate.

(with the exception of sample C) were higher than $1,500 \mathrm{mg} / 100 \mathrm{~g}$ and $330 \mathrm{mg} / 100 \mathrm{~g}$, whereas those of teas costing less than $\$ 25$ were less than $700 \mathrm{mg} / 100 \mathrm{~g}$ and $100 \mathrm{mg} / 100 \mathrm{~g}$, respectively.

\section{Discussion}

In Japan, the color of matcha is a very important factor for judging quality, and higher-quality matcha teas show lower $\mathrm{a}^{*}$ values in the La*b* color space, corresponding to a stronger green color (Kohata et al. 2001a). Matcha teas also show a higher chlorophyll content than other varieties of green tea in Japan (Kohata et al. 1999). Considering the analysis of powdered green teas collected in the US (most of which are labeled as "matcha"), the more expensive teas showed higher levels of chlorophylls, as observed in Japan (Fig. 1).

The conversion percentage of chlorophyll to pheophytin is considered an indicator of the deteriorated color of tea after the production process of raw tea leaves. Takashima et al. (2000) showed that the conversion percentage increased with steaming time during the production process of sencha. Tanaka et al. (1972) showed that it also increased due to the improper storage of sencha. As the production and distribution systems of tea vary across countries, it would be interesting to compare the conversion percentages for the countries of production. The conversion percentages noted for Japan and other countries are as shown

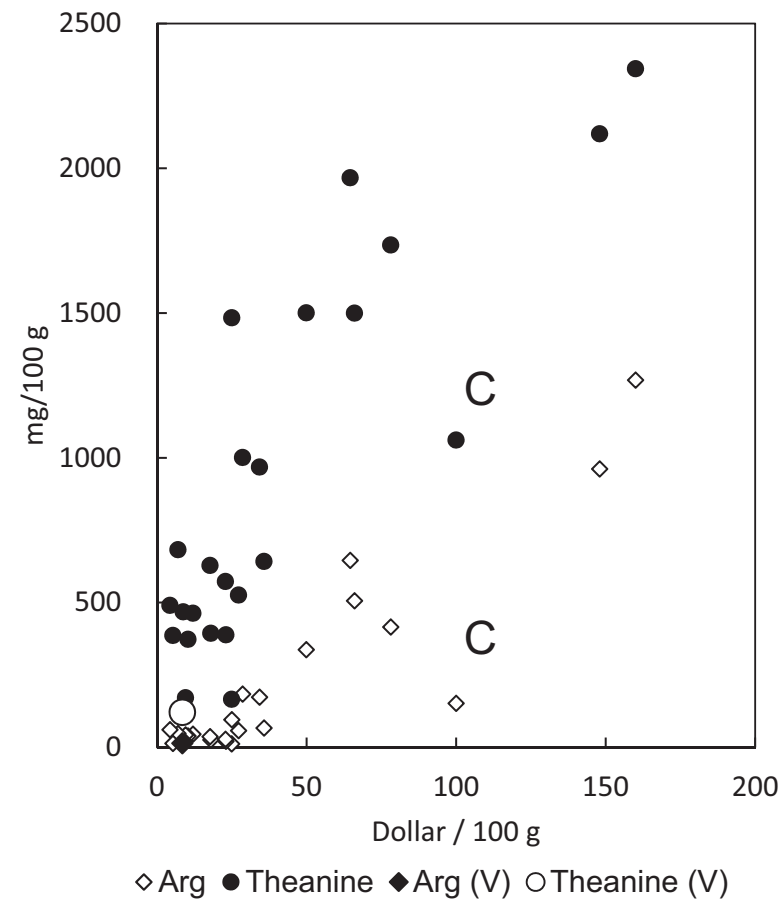

Fig. 4. Theanine and arginine contents in powdered green teas

in Fig. 2. The samples produced in Japan showed a lower conversion percentage than similarly priced samples produced in other countries. The more expensive samples were produced in Japan, where the raw leaves are generally steamed to inactivate the enzymes and then dried using a special drier (called "tencha-ro") to produce high-grade matcha. Kohata et al. (2001b) showed that tea production using a tencha-ro lowered the conversion percentage as compared to production using authentic machines for producing sencha. In other cheaper samples produced in Japan, the inactivation of enzymes is probably achieved using steaming, whereas pan-firing is typically used to inactivate the enzymes in most tea production processes in other countries. Miyazaki et al. (2009) compared the color of pan-fired and steamed teas made from the same batch of raw tea leaves using spectrophotometry, and reported that the green color of pan-fired tea was weaker than that of steamed tea. It is thus supposed that steaming performs better than pan-firing in maintaining chlorophyll levels.

The levels of EGC and EGCG varied between 1.0 and $4.6 \mathrm{~g} / 100 \mathrm{~g}$ and 5.3 and $9.3 \mathrm{~g} / 100 \mathrm{~g}$, respectively, in all samples. However, these levels in samples costing more than $\$ 50$ varied between 1.0 and $2.3 \mathrm{~g} / 100 \mathrm{~g}$ and 5.9 and $7.1 \mathrm{~g} / 100 \mathrm{~g}$, respectively (Fig. 3), with one exception (sample C). The content of catechins showed a close distribution in the more expensive samples. Since the expensive samples were produced in Japan as matcha for use in tea ceremonies, the cultivars and cultivation were similar among 
these teas, as reflected in the similar content of catechins. Conversely, the catechin content of teas costing less than $\$ 30$ per $100 \mathrm{~g}$ varied widely. Because these teas were produced in multiple countries, the cultivars, cultivation, and/or processing were believed to be different.

The expensive teas were rich in both theanine and arginine (Fig. 4). Amino acids are related to the umami taste of green teas and reportedly abundant in high-grade matcha (Goto et al. 1996), as was confirmed for the expensive powdered teas sold in the US.

The one exception as discussed above was sample $\mathrm{C}$, labeled as "matcha" for tea ceremonies. The content of chlorophylls and that of amino acids in sample $\mathrm{C}$ were less than those of similarly priced teas, while the EGC content was higher, thereby indicating that this tea was lower in quality than matcha of similar prices. This tea was made in Japan and labeled as an organic tea. There have been other examples of organic ceremonial-grade matcha of inferior quality sold in the Japanese market (unpublished data). The production of organic tea is rare in Japan. Given the limited availability of organic tea, some organic teas produced in Japan are consequently sold at higher prices relative to the quality.

In this research, we compared the chemical components of powdered teas collected in the US. It was found that powdered teas with a price of less than $\$ 5$ per $100 \mathrm{~g}$ were sold as matcha teas in the US, and that the chemical components of such teas were very different from those of the authentic matcha imported from Japan for use in traditional tea ceremonies. It is thus necessary to define matcha and subsequently disseminate this information to the world, in order to share the status of matcha as a specialty tea, and not just a powdered tea. For this purpose, we are now gathering and analyzing matcha and powdered green tea samples produced in Japan and other countries.

\section{References}

Goto, T. et al. (1996) Chemical composition of commercially available Japanese green tea. Food \& Food Ingredients Journal of Japan, 170, 46-52.

Haraguchi, Y. et al. (2002) Effects of storage conditions on quality of matcha. Chagyo kenkyu houkoku (Tea Res. J.), 93, 1-8 [In Japanese with English summary].

Horie, H. (2012) Production of guanylic acid by heating vegetables. Nippon chori kagaku kaishi (Journal of Cookery Science of Japan), 45, 346-351 [In Japanese with English summary].

Kohata, K. et al. (1998) High performance liquid chromatographic determination of pheophorbide-a and its related chlorophyll derivatives in tea leaves. Food Sci. Technol. Int. Tokyo, 4, 80-84.

Kohata, K. et al. (1999) Investigations of qualities and properties of commercially available green teas on the basis of chlorophyll and its derivatives contents. Chagyo kenkyu houkoku (Tea Res. J.), 87, 13-19 [In Japanese with English summary].

Kohata, K. et al. (2001a) Appearance color measurement of commercially available green teas and application to evaluation of tea quality using a color difference meter. Yasaichagyo shikenjo houkoku (Bull. Natl. Res. Inst. Veg., Ornam. Plants \& Tea) 16, 9-18 [In Japanese with English summary].

Kohata, K. et al. (2001b) Changes in contents of chlorophyll pigments and chlollophylase activity during manufacturing of Tencha. Nippon shokuhin kagaku kogaku kaishi (Journal of the Japanese Society for Food Science and Technology), 48, 744-750 [In Japanese with English summary].

Kuwabara, H. et al. (2016) O-Matcha no subete (All about matcha). Cha (The Tea), 69 (1), 24-29 [In Japanese].

Maeda-Yamamoto, M. et al. (2004) Changes in epigallocatechin3-O-(3-O-methyl) gallate and strictinin contents of tea (Camellia sinensis L.) cultivar 'Benifuki' in various degrees of maturity and leaf order. Food Science and Technology Research, 10, 186-190.

Matsunaga, A. et al. (2016) Effects of various directly covered shading levels on chemical components in tea new shoots of the first flush. Chagyo kenkyu houkoku (Tea Res. J.), 122, 1-7. [In Japanese with English summary].

Miyazaki, H. et al. (2009) Characteristic analysis by the spectro photometer for Kamairicha (pan fired tea) and Tamaryokucha (steamed tea). Chagyo kenkyu houkoku (Tea Res. $J$.), 107, 51-60 [In Japanese with English summary].

Takashima, K. et al. (2000) Properties of the green tea cultivar 'Sakimidori' based on chlorophyll and pheophytin contents. Chagyo kenkyu houkoku (Tea Res. J.), 89, 15-21 [In Japanese with English summary].

Tanaka, S. \& Hara, T. (1972) Changes of chlorophyll in green tea during storage. Chagyo gijutsu kenkyu (Study of Tea), 44, 25-30 [In Japanese with English summary]. 